
JNM
J Neurogastroenterol Motil, Vol. 28 No. 1 January, 2022
pISSN: 2093-0879 elSSN: 2093-0887
https://doi.org/10.5056/jnm20138

\title{
Onigiri Esophagography as a Screening Test for Esophageal Motility Disorders
}

\author{
Shohei Hamada, ${ }^{1}$ Eikichi Ihara, ${ }^{1,2 *}$ Kazumasa Muta, ${ }^{1}$ Masafumi Wada, ${ }^{1}$ Yoshitaka Hata, ${ }^{1}$ Hiroko Ikeda, ${ }^{1}$ Yoshimasa Tanaka, \\ Haruei Ogino, ${ }^{1}$ Takatoshi Chinen, ${ }^{1}$ and Yoshihiro Ogawa ${ }^{1}$ \\ Departments of ${ }^{1}$ Medicine and Bioregulatory Science and ${ }^{2}$ Gastroenterology and Metabolism, Graduate School of Medical Sciences, \\ Kyushu University, Maidashi, Higashi-ku, Fukuoka, Japan
}

\section{Background/Aims}

No screening test for esophageal motility disorder (EMD) has been established, the objective of this study is to examine the potential usefulness of our newly developed "Onigiri esophagography" combined with an obstruction level (OL) classification system in screening for EMD.

\section{Methods}

A total of 102 patients with suspected EMDs who underwent both high-resolution manometry (HRM) and Onigiri esophagography between April 2017 and January 2019 were examined. The EMD diagnosis was performed based on the Chicago classification version 3.0 by HRM. Onigiri esophagography was performed using a liquid medium (barium sulfate) followed by a solid medium, which consisted of an Onigiri (a Japanese rice ball) with barium powder. The extent of medium obstruction was assessed by the OL classification, which was defined in a stepwise fashion from OLO (no obstruction) to OL4 (severe obstruction).

\section{Results}

The patients with OLO (32.3\%), OL1 (50.0\%), OL2 (88.0\%), OL3 (100.0\%), and OL4 (100.0\%) were diagnosed EMDs by HRM. The area under the curve, as determined by a receiver operating characteristic analysis, for the OL classification was 0.86 . Using the cutoff value of OL1, the sensitivity and specificity were $87.3 \%$ and $61.3 \%$, respectively, while using a cutoff value of OL2, the sensitivity and specificity were $73.2 \%$ and $90.3 \%$, respectively.

\section{Conclusion}

In conclusion, Onigiri esophagography combined with the OL classification system can be used as a screening test for EMDs with a cutoff value of OL1.

(J Neurogastroenterol Motil 2022;28:43-52)

\section{Key Words}

Barium sulfate; Esophageal motility disorders; Manometry

Received: June 17, 2020 Revised: September 13, 2020 Accepted: November 10, 2020

(a) This is an Open Access article distributed under the terms of the Creative Commons Attribution Non-Commercial License (http://creativecommons. org/licenses/by-nc/4.0) which permits unrestricted non-commercial use, distribution, and reproduction in any medium, provided the original work is properly cited.

${ }^{*}$ Correspondence: Eikichi Ihara, MD, PhD

Department of Gastroenterology and Metabolism, Graduate School of Medical Sciences, Kyushu University, 3-1-1 Maidashi, Higashi-ku, Fukuoka 812-8582, Japan

Tel: +81-92-642-5286, Fax: +81-92-642-5287, E-mail: eikichi@intmed3.med.kyushu-u.ac.jp 


\section{Introduction}

Meal intake, which is initiated by the swallowing of food, is critical to life. Esophageal motility is responsible for the third phase of swallowing, and major dysfunction of esophageal motility is referred to as esophageal motility disorder (EMD), which is characterized by difficulty in swallowing, chest pain, and reflux symptoms. ${ }^{1}$ The recent development of high-resolution manometry (HRM), together with the Chicago classification (CC) version 3.0 (v3.0), has allowed us to establish a diagnostic system for EMDs. ${ }^{2}$

Although it is clear that HRM together with CC v3.0 is the gold standard for the diagnosis of EMDs, it cannot be applied to patients widely or globally in general clinical practice, as HRM systems are costly to install and maintain. ${ }^{3,4}$ Thus, certain diagnostic tools for screening EMDs are required. It was reported that the conventionally-performed barium esophagography is useful for detecting EMDs by evaluating the extent of esophageal obstruction in relation to fluids. ${ }^{5,6}$ However, all the EMDs other than the most severe EMD, namely achalasia does not usually cause stagnation of liquid barium. The sensitivity and specificity of esophagography using liquid barium in screening for EMDs were not sufficient. ${ }^{7,8}$

In this study, we propose a new diagnostic method; "Onigiri esophagography" combined with an obstruction level (OL) classification system as a screening test for EMD. Onigiri is a Japanese rice ball, which, when combined with barium powder, was used as a solid medium for esophagography. The OL classification system is our newly proposed index of liquid and solid obstruction. The objective of this study is to determine the usefulness of Onigiri esophagography combined with the OL classification system in screening for EMDs.

\section{Materials and Methods}

\section{Patients and Methods}

Both HRM and Onigiri esophagography were applied to patients with suspected esophageal motility disorders due to the presence of persistent symptoms, including heartburn, chest pain, chest discomfort, and dysphagia, which were refractory to proton pump inhibitors, despite normal esophagus esophagogastroduodenoscopy results at Kyushu University Hospital. Between April 2017 and January 2019, a total of 106 patients with suspected EMDs were enrolled in this study (Fig. 1A). Four cases with extramural compression to a level of $\leq 5$ in the short axis of the esophagus were excluded. As a result, 102 patients were analyzed in this study. The Eckardt score ${ }^{9}$ was obtained for each patient using a questionnaire. This research complied with the guidelines for human studies and should include evidence that the research was conducted ethically in accordance with the World Medical Association Declaration of Helsinki. The subjects in this study gave their written informed consent and this study protocol was approved by Kyushu University Hospital's committee on human research (29-35).

\section{The Onigiri Esophagography Protocol}

Onigiri esophagography was performed and the OL classification was defined as follows, with the procedures performed without the use of any antispasmodic agents. The evaluation of passage through the esophagus was defined as complete esophageal emptying within 10 seconds after swallowing. The patients were asked to swallow $10 \mathrm{~mL}$ of barium sulfate $(100 \mathrm{w} / \mathrm{v} \%$; Barytester A240; FUSHIMI Pharmaceutical Co, Kagawa, Japan) in an upright position. Esophagography was performed in the right anterior and posterior oblique view to visualize the passage of medium using a fluoroscope (DREX-ULT80/07; Toshiba, Tokyo, Japan). When liquid barium sulfate remained in the esophagus, forming the air
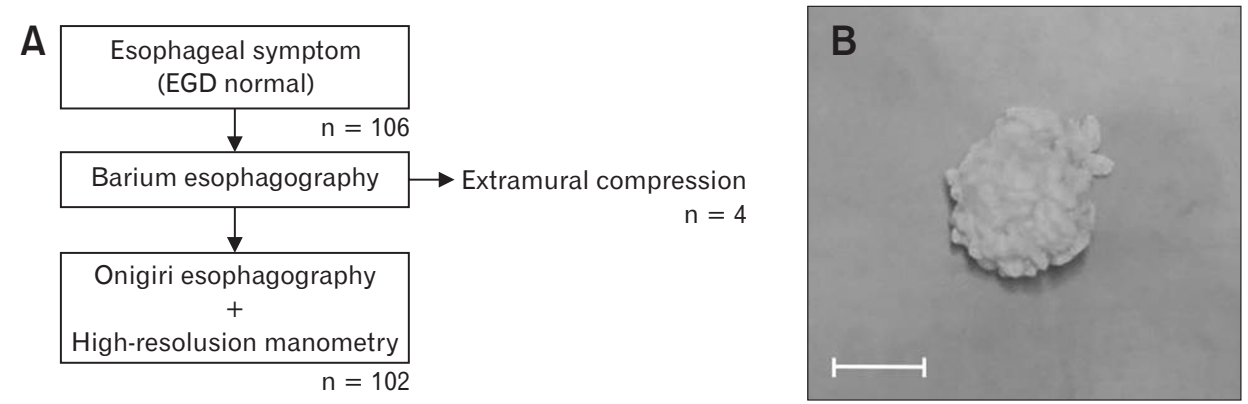

Figure 1. A flow chart of this study (A) and a $10 \mathrm{~g}$ Onigiri with barium powder (B). Barium powder was attached exclusively to the surface of the Onigiri. The bar indicates $2 \mathrm{~cm}$. EGD, esophagogastroduodenoscopy. 
fluid level, the condition was defined as OL4 (Fig. 2B). When the successful passage of barium medium was obtained (Fig. 2A), the patient underwent the next procedure using the solid contents of an Onigiri with barium powder. The patient was asked to chew a $10-\mathrm{g}$ Onigiri with barium powder 15 times (Fig. 1B) and then swallow. When solid contents passed successfully into the stomach without any further action, it was defined as OL0 (Fig. 2C). When the solid contents remained in the esophagus, the patient was asked to first perform a dry swallow several times and, if required, to swallow $10 \mathrm{~mL}$ of barium. OL1 was defined as successful passage of the remaining solid contents obtained by the dry swallow (Fig. 2D), and OL2 was defined as successful passage obtained by swallowing $10 \mathrm{~mL}$ of liquid barium (Fig. 2E). Finally, OL 3 was defined by the solid contents remaining in the esophagus after the swallowing of $10 \mathrm{~mL}$ of liquid barium (Fig. 2F). The abovementioned procedure was decided by the agreement of 3 gastrointestinal (GI) experts. If their opinions differed, it was decided through discussion, and it was repeated 3 times to determine the final OL level of the patient to be more frequent results. If the final OL level was not determined within 3 evaluations, further evaluations were added until the final OL level was determined (Fig. 3A)

\section{The Onigiri Esophagography Video Test}

The inter-observer agreement of Onigiri esophagography among the examinees was determined by calculating Fleiss' kappa coefficient (described later) using an Onigiri esophagography video test, which was created from videos of the cases enrolled in this study. Six examinees, including 3 GI experts who were Onigiri esophagography examiners and 3 GI trainees who had never been involved in Onigiri esophagography were enrolled. The Onigiri
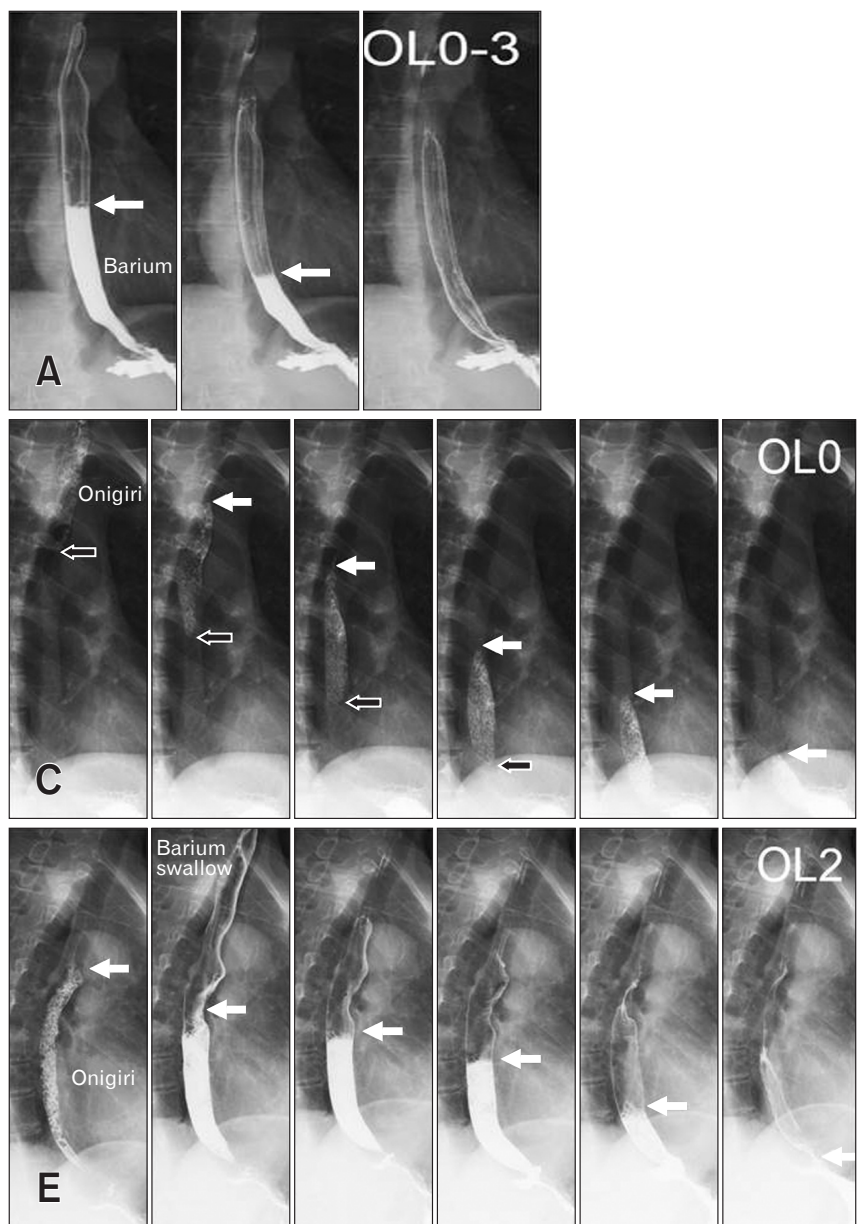
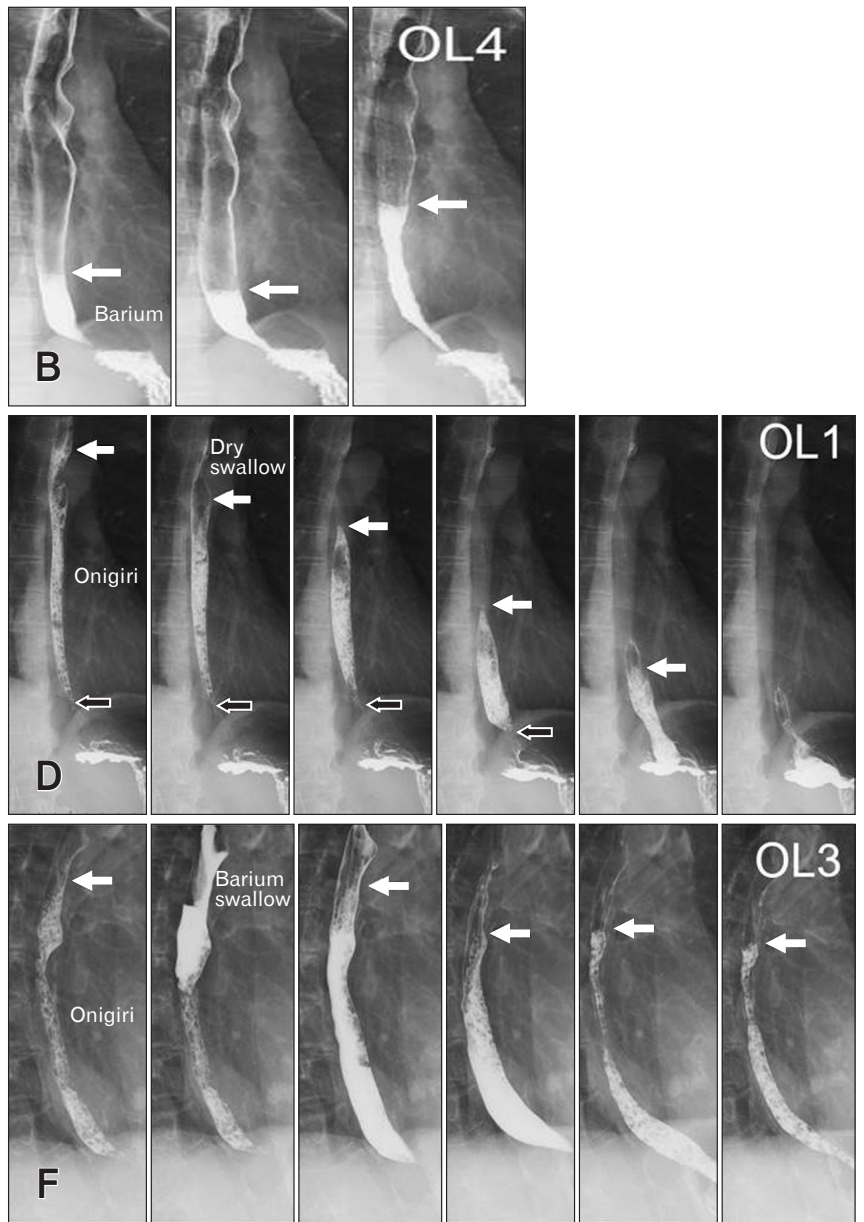

Figure 2. Onigiri esophagography combined with the obstruction level (OL) classification system. (A, B) A representative esophagography series with $10 \mathrm{~mL}$ of liquid barium for OL0, 1, 2, and 3 (A) and for OL4 (B) is shown. White arrow indicates the upper level of the swallowed liquid barium. (C-F) A representative Onigiri esophagography series for OL0 (C), OL1 (D), OL2 (E), and OL3 (F) is shown. White arrows indicate the upper level of swallowed barium with an Onigiri. Black arrows indicate the lower level of swallowed barium with an Onigiri. 


\section{A}
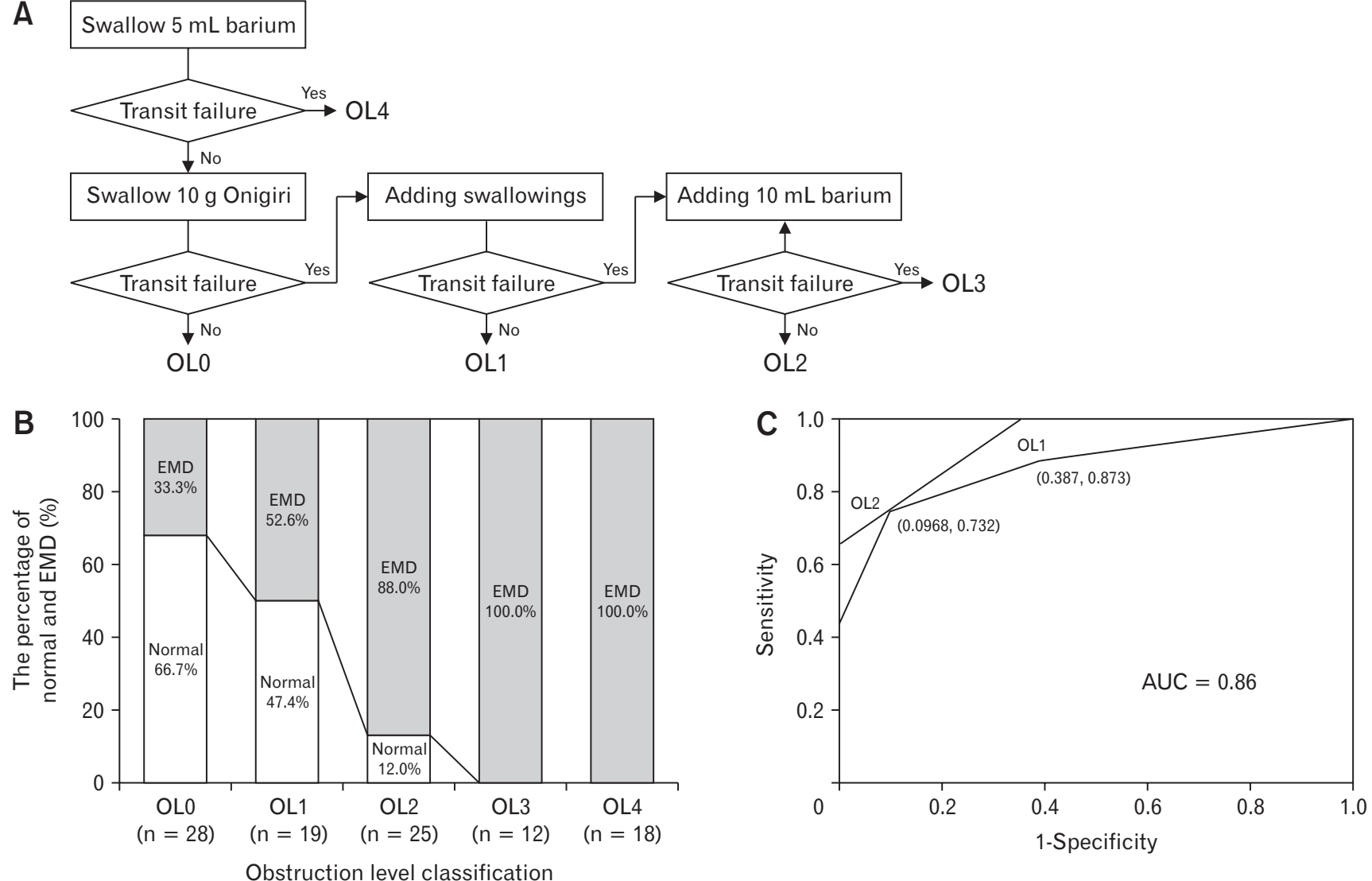

Figure 3. Onigiri esophagography combined with the obstruction level (OL) classification system as a screening test for esophageal motility disorders. (A) A flow chart of Onigiri esophagography to determine the OL classification score. (B) The proportion of esophageal motility disorders (EMDs) in each OL classification. (C) The receiver operating characteristic analyses for the OL classification. AUC, area under the curve.

esophagography video test consisted of 4 patterns of video clips ( $\mathrm{n}$ $=40$ ), which were necessary and sufficient to determine each obstruction level, including (1) swallowing $10 \mathrm{~mL}$ of liquid barium (n $=10)$, (2) swallowing $10 \mathrm{~g}$ of Onigiri $(\mathrm{n}=10)$, (3) additional dry swallowing of the remaining Onigiri $(n=10)$, and (4) swallowing of an additional $10 \mathrm{~mL}$ of liquid barium $(\mathrm{n}=10)$ for the remaining Onigiri. The examinees were asked to judge if liquid barium or Onigiri successfully passed through the esophagus.

\section{The High-resolution Manometry Protocol}

The esophageal motility function was assessed by HRM using a Manoscan Z (Given Imaging, Los Angeles, CA, USA). HRM was performed using a standardized protocol. In brief, the patients were asked to perform 10 swallows of $5 \mathrm{~mL}$ of water in the supine position. ${ }^{2}$ After the recording the basal condition without any swallows, the subjects were instructed to swallow as infrequently as possible and to breathe quietly and regularly. HRM data based on 10 swallows were analyzed using ManoView ESO 3.0 (Given Imag- ing). The HRM diagnosis was performed based on the $\mathrm{CC}$ v3.0. ${ }^{2}$ The parameters of HRM are shown as the mean of 10 swallows.

\section{Statistical Methods}

All statistical analyses were performed using the JMP Pro 14.2.0 software program (SAS Institute, Cary, NC, USA). The data were expressed as the median (interquartile range). Pearson's chi-squared test was used to analyze the categorical data, while Mann-Whitney's $U$ test was used to analyze the continuous and ordinal data. The relationship between different pairs was assessed using Spearman's rank correlation. Receiver operating characteristic (ROC) analyses were conducted to identify a more accurate cutoff point that could help identify the probability of EMD. ROC curves were created by plotting the range of sensitivity and specificity pairs for each participant's EMD rate. A global assessment of the performance of the test was given performed based on the area under the ROC curve. Fleiss' kappa coefficient was calculated in the interobserver reliability test. The kappa value was expressed as $\kappa(95 \%$ 
confidence interval), which was interpreted using the Landis's scale (values of 0.00-0.20 represent poor agreement, 0.21-0.40 represent slight agreement, 0.41-0.60 represent moderate agreement, 0.600.80 represent substantial agreement, and 0.81-1.00 represent almost perfect agreement). ${ }^{10} P$-values of $<0.05$ were considered to indicate statistical significance.

\section{Results}

\section{Clinical Characteristics of the Patients Enrolled in This Study}

The clinical characteristics of the patients enrolled in this study are shown in Table. The median age, body mass index, and Eckardt score were 61 (51.0-72.3) years, $21.8(19.3-24.6) \mathrm{kg} / \mathrm{m}^{2}$, and 3 (2$5)$, respectively. The study population included 55 females and 47 males. Based on CC v3.0, the HRM studies indicated the following findings in the total study population of 102 patients: those with achalasia ( $\mathrm{n}=15$; type I/type II/type III: $1 / 11 / 3$ ), esophagogastric junction outflow obstruction (EGJOO; $n=22$ ), absent contractility $(\mathrm{n}=11)$, distal esophageal spasm (DES; $\mathrm{n}=2)$, jackhammer esophagus $(\mathrm{n}=6)$, ineffective esophageal motility (IEM; $\mathrm{n}=13$ ), fragmented peristalsis $(\mathrm{n}=2)$, and normal HRM study results $(\mathrm{n}=31)$. No adverse events occurred in the 2 tests in this study.

Table. Summary of the 102 Patients Who Underwent High-resolution Manometry

\begin{tabular}{lc}
\hline \multicolumn{1}{c}{ Clinical characteristics } & Median (IQR) or n \\
\hline Age $(\mathrm{yr})$ & $61.0(51.0-72.3)$ \\
Sex $(\mathrm{female} / \mathrm{male})$ & $55 / 47$ \\
Body mass index $\left(\mathrm{kg} / \mathrm{m}^{2}\right)$ & $21.8(19.3-24.6)$ \\
Eckardt score & $3(2-5)$ \\
HRM diagnosis & 1 \\
Type I achalasia & 11 \\
Type II achalasia & 3 \\
Type III achalasia & 22 \\
EGJ outflow obstruction & 11 \\
Absent contractility & 2 \\
Distal esophageal spasm & 6 \\
Jackhammer esophagus & 13 \\
Ineffective esophageal motility & 2 \\
Fragmented peristalsis & 31 \\
Normal & \\
\hline
\end{tabular}

IQR, interquartile range; HRM, high-resolution manometry; EGJ, esophagogastric junction.

\section{The Proportion of Esophageal Motility Disorder Diagnosis in Patients With Each Obstruction Level Value}

According to the results of Onigiri esophagography, the 102 patients were classified as follows OL0, $\mathrm{n}=28$; OL1, $\mathrm{n}=19$; OL2, $n=25 ;$ OL $3, n=12$; and OL4, $n=18$. The patients with OL0 (32.3\%), OL1 (50.0\%), OL2 (87.0\%), OL3 (100.0\%), and OL4 (100.0\%) were diagnosed with EMDs by the HRM studies; that is, all the HRM diagnosis other than normal HRM study (Fig. 3B). The area under the curve, as determined by a ROC analysis, for the OL classification was 0.86 . Using the cutoff value of OL1, the sensitivity and specificity were $87.3 \%$ and $61.3 \%$ while positive and negative predictive values were $83.8 \%$ and $67.9 \%$, respectively. Using the cut off value of OL2, the sensitivity and specificity were $73.2 \%$ and $90.3 \%$ while positive and negative predictive values were $94.5 \%$ and $60.9 \%$, respectively (Fig. 3C). The Eckardt scores for OL0, OL1, OL2, OL3, and OL4 were 2.0 (1.0-4.0), 3.0 (2.0-5.0), $3.0(2.0-4.5), 3.0(2.0-5.5)$, and $5.0(2.0-7.3)$, respectively.

\section{The Obstruction Level Value of the Obstruction Level Classification Correlated With the Eckardt Score and Each High-resolution Manometry Metrics}

The Eckardt scores for OL0, OL1, OL2, OL3, and OL4 were 2.0 (1.0-4.0), 3.0 (2.0-5.0), 3.0 (2.0-4.5), 3.0 (2.0-5.5), and 5.0 (2.0-7.3), respectively. There was a significant positive correlation between the OL value of the OL classification and the Eckardt score (Spearman $\rho=0.24, P<0.05$ ) (Fig. 4A). As for HRM metrics, there was a significant positive correlation between the OL value of the $\mathrm{OL}$ classification and the integrated relaxation pressure (Spearman $\rho=0.35, P<0.01$ ) (Fig. 4B). In contrast, there was a negative correlation between the OL value of the OL classification and distal contractile integral $(n=91$, Spearman $\rho=-0.40$, $P<0.01$; Fig. 4 C); patients with type 2 achalasia $(\mathrm{n}=11)$ were excluded from this analysis since distal contractile integral could not be assessed in these patients. Furthermore, there was also a negative correlation between the OL value of the OL classification and distal latency $(\mathrm{n}=78$, Spearman $\rho=-0.30, P<0.01$; Fig. 4D); patients with type I achalasia $(n=1)$, type II achalasia $(n=11)$, or absent contractility $(n=11)$ were excluded from this analysis since distal latency could not be assessed in these patients. 

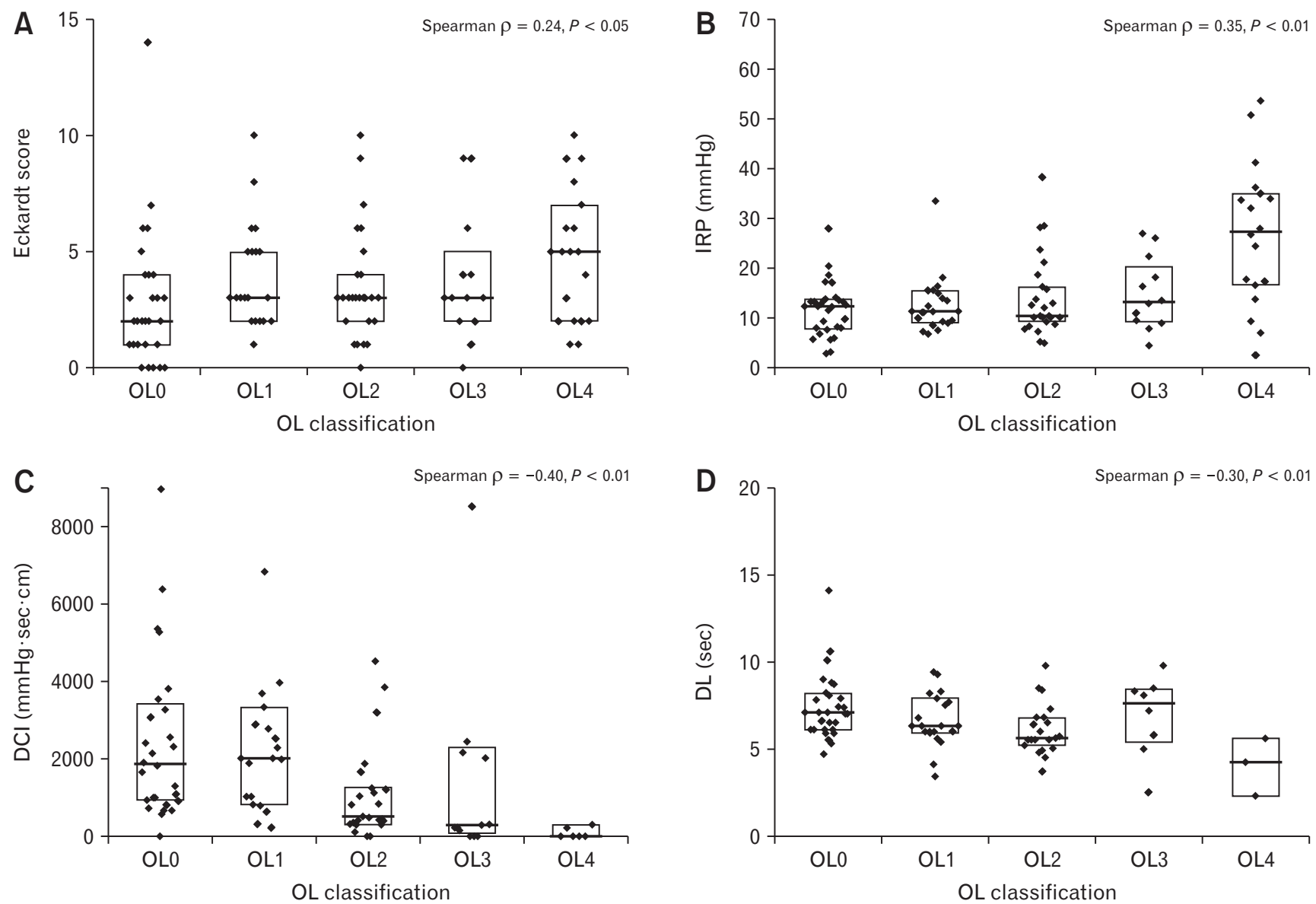

Figure 4. Correlations between the obstruction level (OL) value of the OL classification and Eckardt score/each high-resolution manometry (HRM) metrics. (A) A correlation between the OL value of the OL classification and the Eckardt score. (B-D) Correlations between the OL value of the OL classification and the HRM metrics including integrated relaxation pressure (IRP) (B), DCI (distal contractile integral) (C), and DL (distal latency) (D).

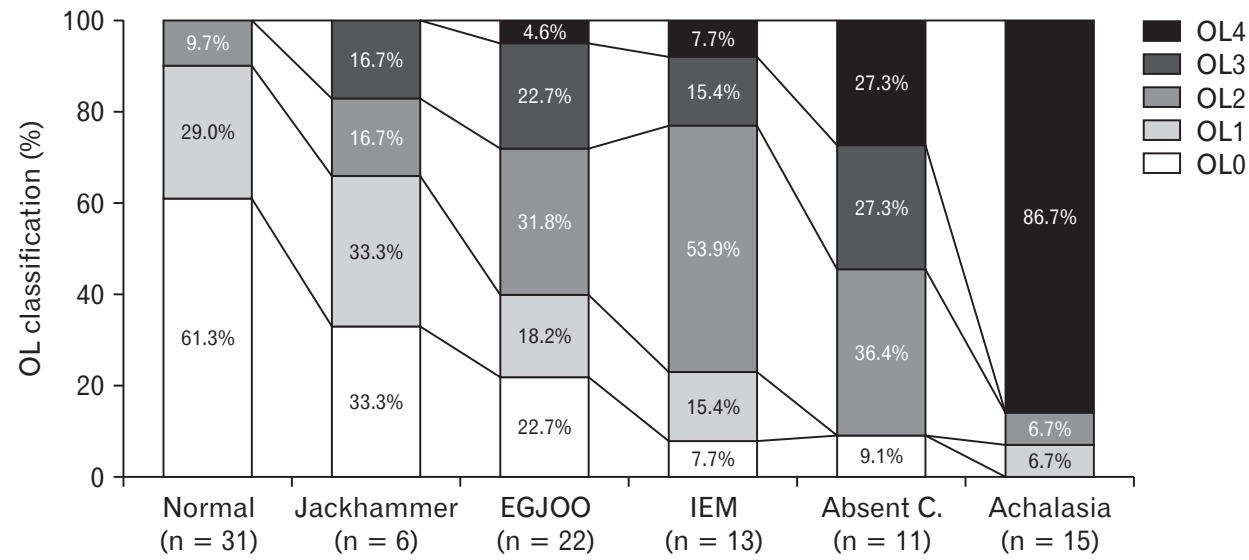

Figure 5. The proportion of obstruction level (OL) classification scores in each esophageal motility disorder. EGJOO, esophagogastric junction outflow obstruction; IEM, ineffective motility; Absent C., absent contractility. 


\section{The Proportion of Obstruction Level Classifications in Patients With Each High-resolution Manometry Diagnosis}

Next, we examined the proportion of OL classification in each HRM diagnosis (Fig. 5). Thirty-one patients with a normal HRM study were classified as follows: OL0 $(n=19[61.3 \%])$, OL1 $(\mathrm{n}=9[29.0 \%])$, OL2 $(\mathrm{n}=3[9.7 \%])$, OL3 $(\mathrm{n}=0$ $[0.0 \%])$, and OL4 $(n=0[0.0 \%])$. Six patients with jackhammer esophagus were classified as follows: OL0 $(n=2[33.3 \%])$, OL1 $(n=2[33.3 \%])$, OL2 $(n=1[16.7 \%])$, OL3 $(n=1$ $[16.7 \%])$, and OL4 $(n=0[0.0 \%])$. Twenty-two patients with EGJOO were classified as follows OL0 $(\mathrm{n}=5[22.7 \%])$, OL1 $(\mathrm{n}=4[18.2 \%])$, OL2 $(\mathrm{n}=7[31.8 \%])$, OL3 $(\mathrm{n}=5[22.7 \%])$, and OL4 ( $\mathrm{n}=1[4.6 \%])$. Thirteen patients with IEM were classified as follows: OL0 $(\mathrm{n}=1[7.7 \%])$, OL1 $(\mathrm{n}=2[15.4 \%])$, OL2 $(n=7[53.9 \%])$, OL3 $(n=2[15.4 \%])$, and OL4 $(n=1$ $[7.7 \%])$. Eleven patients with absent contractility were classified as follows: OL0 ( $\mathrm{n}=1[9.1 \%])$, OL1 ( $\mathrm{n}=0[0.0 \%])$, OL2 ( $\mathrm{n}=$ $4[36.4 \%])$, OL3 $(n=3[27.3 \%])$, and OL4 $(n=3[27.3 \%])$. Finally, fifteen patients with achalasia were classified as follows; $\operatorname{OL} 0(n=0[0.0 \%]), \operatorname{OL} 1(n=1[6.7 \%])$, OL2 $(n=1[6.7 \%])$, OL3 $(n=0[0.0 \%])$, and OL4 $(n=13[86.7 \%])$, where both patients with OL1 and OL2 were diagnosed with type III achalasia. Although the number of the patients with DES $(n=2)$ and fragmented peristalsis $(n=2)$ was small, DES and fragmented peristalsis both included 1 patient each with OL2 and OL3.

\section{Results of the Onigiri Esophagography Video Test}

Substantial agreement $(\kappa=0.77,0.73-0.81)$ was obtained in a total of 6 examinees, where the $\kappa$ values for the 3 GI experts and 3 GI trainees were 0.87 (0.77-0.96, almost perfect agreement) and 0.77 (0.67-0.86, substantial agreement), respectively.

At least 1 examinee made a different judgment from the others in 10 of 40 video clips, which included (1) swallowing $5 \mathrm{~mL}$ of liquid barium ( $\mathrm{n}=6$ ), (2) swallowing $10 \mathrm{~g}$ of Onigiri $(\mathrm{n}=0)$, (3) additional dry swallowing of the remaining Onigiri $(\mathrm{n}=2)$, and (4) swallowing an additional $10 \mathrm{~mL}$ of liquid barium for the remaining Onigiri $(n=2)$. This means that complete agreement could be obtained in the remaining 30 video clips.

\section{Discussion}

EMDs have a significant impact on society, since they reduce not only quality of life and social labor productivity due to difficulty in swallowing, chest pain and reflux symptoms, ${ }^{1,11-13}$ but can also lead to life-threatening diseases, such as aspiration pneumonia. ${ }^{14} \mathrm{It}$ has been considerably difficult to detect and diagnose EMDs due to the functional nature of these diseases. ${ }^{15}$ More recently, however, based on HRM with CC v3.0, there have been major developments in the medical practice for EMDs. The major EMDs are classified into achalasia, EGJOO, DES, jackhammer esophagus, and absent contractility, among which EGJOO is a newly established concept of disorder defined as impaired lower esophageal sphincter (LES) relaxation with intact esophageal body peristalsis, while achalasia is characterized by both impaired LES relaxation and esophageal body peristalsis. ${ }^{2,16}$ In addition to the major EMDs, IEM and fragmented peristalsis are defined as minor EMDs, and their real clinical significance and appropriate managements are still under investigation. ${ }^{17,18}$ Despite the drastic development in diagnostic methods for EMD, in community healthcare, the medical practice in relation to EMDs is still challenging, since HRM is not usually available at local medical institutions. Thus, development of simple screening tests for EMDs is required to facilitate the referral of patients with suspected EMDs to specialists. For this purpose, we have proposed Onigiri esophagography combined with the OL classification system as a screening test for EMDs. There have been several studies to evaluate EMDs using solid meals, however, most of these were conducted in combination with HRM; they were rarely conducted with esophagography. It has been shown that the diagnostic sensitivity of HRM using a solid meal for EMDs was high, compared to HRM using a liquid meal, ${ }^{19-21}$ indicating that esophageal motility dysfunction was evoked by a solid meal more frequently than by a liquid meal. These findings support the validity of Onigiri esophagography in the present study. As a result, in this study, we demonstrated that Onigiri esophagography combined with the OL classification system was a highly sensitive test with a cutoff value of OL1 and a highly specific test with a cutoff value of OL2. Importantly, the OL classification score of Onigiri esophagography was positively associated with esophageal symptoms.

One previous study examined the usefulness of liquid barium esophagography as a screening test for EMDs in comparison to $\mathrm{HRM}^{7}$ The sensitivity and specificity of barium esophagography in the detection of EMDs was $69.0 \%$ and $50.0 \%$, respectively, which was low. ${ }^{7}$ They concluded that barium esophagography was useful in the assessment of anatomic abnormalities but is a poor screening examination for the detection of EMDs. Another study which only recruited patients with achalasia reported that the sensitivity of barium esophagography in the diagnosis of achalasia was $78.3 \%$, which was still not enough. ${ }^{6}$ We have thought of the pos- 
sible usefulness of a solid meal, specifically the Onigiri. Recently, a number of studies have been carried out to examine the usefulness of HRM using solid meals rather than a liquid medium, and the Onigiri has been shown to be one of the best candidate solid mediums. ${ }^{22-26}$ It seems that the viscosity and shape of the Onigiri is appropriate for examining the esophageal motility function. Furthermore, one advantage of using an Onigiri-which remains to be addressed - is that the viscosity and shape of the Onigiri can be adjusted by adding a certain amount of barium liquid. As a result, in comparison to previous studies using liquid barium esophagography, Onigiri esophagography with the OL classification system could achieve high sensitivity (87.3\%) with a cutoff value of OL1, and high specificity $(90.3 \%)$ with a cutoff value of OL2. Therefore, Onigiri esophagography with the OL classification system and a cutoff value of OL1 can be used as a screening test to detect EMDs in local medical institutions.

It is important to pursue the possibility of further improving the sensitivity of Onigiri esophagography with the OL classification system. One factor that reduced the sensitivity for OL1 in this study was that $33.3 \%$ of the patents with OL0 and $52.6 \%$ of the patients with OL1 still had EMDs. The esophageal motility function consists of 2 major mechanisms: esophageal body peristalsis, which is responsible for esophageal clearance, and basal LES contraction with transient relaxation during the swallowing action. ${ }^{27}$ Among the EMDs based on $\mathrm{CC}$ v3.0, esophageal body peristalsis is retained in patients with EGJOO and is also retained in most patients with jackhammer esophagus. Indeed, $40.9 \%$ of the patients with EGJOO and $66.6 \%$ of those with jackhammer esophagus exhibited OL0 or OL1 (Fig. 5). Therefore, Onigiri esophagography combined with the OL classification system is more suitable for the detection of disorders with impaired esophageal body clearance, including achalasia, absent contractility, and ineffective motility, as opposed to those with intact esophageal body peristalsis, such as EGJOO and jackhammer esophagus. High OL scores in IEM and absent contractility indicate that normal esophageal body peristalsis plays a role in transporting ingested solid food to the esophagus. Although the sensitivity of Onigiri esophagography may be improved by incorporating the time of esophageal clearance into this system, we did not adopt this parameter in the present study. In the present study, we focused on simplifying the diagnostic method as much as possible, to make it suitable for non-GI specialist doctors to judge. In contrast, the reduction in specificity may be partly because $47.4 \%$ of the patients with OL1 and $12.0 \%$ of the patients with OL2 had normal HRM results. Considering that the OL classification score of Onigiri esophagography was positively associated with esopha- geal symptoms, it may be necessary to adjust the diagnostic criteria for a normal HRM study.

It has been reported that the specific X-ray findings of liquid barium esophagography, such as corkscrew appearance, beaklike narrowing of the distal esophagus, and failed peristalsis contributed to the detection of EMDs. ${ }^{28,29}$ We adopted Onigiri esophagography with OL classification from a viewpoint that it is a method that can be widely used by non-specialists to screen for EMDs at local medical institutions. Indeed, substantial agreement $(\kappa=0.77,0.73-0.81)$ was obtained among a total of 6 examinees including not only GI experts but also GI trainees. In this study, judgement of the successful passage in video clips with liquid barium showing "swallowing 5 $\mathrm{mL}$ of liquid barium" was low in comparison to that in video clips with Onigiri, which showed "swallowing $10 \mathrm{~g}$ of Onigiri," "additional dry swallowing of the remaining Onigiri," and "swallowing an additional $10 \mathrm{~mL}$ of liquid barium for the remaining Onigiri." This may be due to the fact that Onigiri migrated in a cluster while liquid barium tended to be dispersed in the esophagus. Solid Onigiri could contribute to an increase in inter-observer agreement in comparison to liquid barium. A further multicenter prospective study is required to determine whether Onigiri esophagography is useful for the detection of EMDs in other medical institutions.

This study was associated with several limitations. First, it was performed in a single center. Second, patients in whom EMD was suspected based on symptoms were enrolled in this study, while normal subjects without symptoms were not recruited. Third, we could not conduct a sub-analysis to compare the efficacy of Onigiri esophagography to that of liquid esophagography, since liquid barium esophagography was performed as a part of the Onigiri esophagography. Thus, liquid barium esophagography could not be performed independently of Onigiri esophagography. It would be desirable to conduct a multicenter prospective randomized comparative study in the near future.

In conclusion, Onigiri esophagography combined with the OL classification system can be used as a screening test for EMD with a cutoff value of OL1. The patients with positive results could be referred to specialists to make a definite diagnosis of EMDs using HRM.

Financial support: This study was supported in part by the Japan Society for the Promotion of Science KAKENHI (17K09351) and by Smoking Research Foundation (2017G026).

Conflicts of interest: Eikichi Ihara participates in the funded research of Takeda Pharmaceutical Co, Ltd and belongs to an endowed course supported by the companies including Ono Phar- 
maceutical Co, Ltd, Miyarisan Pharmaceutical Co, Ltd, Sanwa Kagaku Kenkyusho Co, Ltd, Otsuka Pharmaceutical Factory, Inc, Fujifilm Medical Co, Ltd, Terumo Corporation, Fancl Corporation, and Ohga Pharmacy. The authors declare no other conflicts of interest in association with this study.

Author contributions: Shohei Hamada, Kazumasa Muta, and Eikichi Ihara designed this study; Shohei Hamada, Kazumasa Muta, Masafumi Wada, and Hiroko Ikeda performed HRM; Yoshitaka Hata and Yoshimasa Tanaka performed EGD; Shohei Hamada and Masafumi Wada collected the data and performed the statistical analysis; Haruei Ogino, Takatoshi Chinen, and Yoshihiro Ogawa provided the technical and material supports; Shohei Hamada and Eikichi Ihara mainly wrote the manuscript; and Takatoshi Chinen and Yoshihiro Ogawa supervised this study.

\section{References}

1. Katz PO, Dalton CB, Richter JE, Wu WC, Castell DO. Esophageal testing of patients with noncardiac chest pain or dysphagia. Results of three years' experience with 1161 patients. Ann Intern Med 1987;106:593-597.

2. Kahrilas PJ, Bredenoord AJ, Fox M, et al. The Chicago classification of esophageal motility disorders, v3.0. Neurogastroenterol Motil 2015;27:160-174.

3. Carroll TL, Werner A, Nahikian K, Dezube A, Roth DF. Rethinking the laryngopharyngeal reflux treatment algorithm: evaluating an alternate empiric dosing regimen and considering up-front, $\mathrm{pH}$-impedance, and manometry testing to minimize cost in treating suspect laryngopharyngeal reflux disease. Laryngoscope 2017;127(suppl 6):S1-S13.

4. Kraft C, Kathpalia P, Baumgardner JM, Pandolfino JE, Sewell JL. How to incorporate esophageal manometry teaching in your fellowship program. Gastroenterology 2019;156:2120-2123.

5. de Oliveira JM, Birgisson S, Doinoff C, et al. Timed barium swallow: a simple technique for evaluating esophageal emptying in patients with achalasia. AJR Am J Roentgenol 1997;169:473-479.

6. Yamasaki T, Tomita T, Mori S, et al. Esophagography in patients with esophageal achalasia diagnosed with high-resolution esophageal manometry. J Neurogastroenterol Motil 2018;24:403-409.

7. O'Rourke AK, Lazar A, Murphy B, Castell DO, Martin-Harris B. Utility of esophagram versus high-resolution manometry in the detection of esophageal dysmotility. Otolaryngol Head Neck Surg 2016;154:888891.

8. Schima W, Stacher G, Pokieser P, et al. Esophageal motor disorders: videofluoroscopic and manometric evaluation--prospective study in 88 symptomatic patients. Radiology 1992;185:487-491.

9. Eckardt VF, Aignherr C, Bernhard G. Predictors of outcome in patients with achalasia treated by pneumatic dilation. Gastroenterology 1992;103:1732-1738.
10. Landis JR, Koch GG. The measurement of observer agreement for categorical data. Biometrics 1977;33:159-174.

11. Schlottmann F, Neto RML, Herbella FAM, Patti MG. Esophageal achalasia: pathophysiology, clinical presentation, and diagnostic evaluation. Am Surg 2018;84:467-472.

12. van Hoeij FB, Ponds FA, Smout AJ, Bredenoord AJ. Incidence and costs of achalasia in The Netherlands. Neurogastroenterol Motil 2018;30:e13195.

13. Hamada $\mathrm{S}$, Ihara $\mathrm{E}$, Ikeda $\mathrm{H}$, et al. Clinical characterization of vonoprazan-refractory gastroesophageal reflux disease. Digestion 2021;102:197204.

14. Gallegos C, Brito-de la Fuente E, Clavé P, Costa A, Assegehegn G. Nutritional aspects of dysphagia management. Adv Food Nutr Res 2017;81:271-318.

15. Frieling T. Non-cardiac chest pain. Visc Med 2018;34:92-96.

16. Muta K, Ihara E, Fukaura K, Tsuchida O, Ochiai T, Nakamura K. Effects of acotiamide on the esophageal motility function in patients with esophageal motility disorders: a pilot study. Digestion 2016;94:9-16.

17. Schlottmann F, Herbella FA, Patti MG. Understanding the Chicago classification: from tracings to patients. J Neurogastroenterol Motil 2017;23:487-494.

18. Balko RA, Codipilly DC, Ravi K. Minor esophageal functional disorders: are they relevant? Curr Treat Options Gastroenterol 2020;18:82-96.

19. Sweis R, Anggiansah A, Wong T, Brady G, Fox M. Assessment of esophageal dysfunction and symptoms during and after a standardized test meal: development and clinical validation of a new methodology utilizing high-resolution manometry. Neurogastroenterol Motil 2014;26:215-228.

20. Ang D, Misselwitz B, Hollenstein M, et al. Diagnostic yield of highresolution manometry with a solid test meal for clinically relevant, symptomatic oesophageal motility disorders: serial diagnostic study. Lancet Gastroenterol Hepatol 2017;2:654-661.

21. Xiang XL, Wang A, Tu L, et al. The motility of esophageal sphincters during liquid and solid bolus swallows: a multicenter normative value study of high-resolution manometry in China. Neurogastroenterol Motil 2017;29:e12914.

22. Hasan Y, Go J, Hashmi SM, Valestin J, Schey R. Influence of everyday bolus consistencies in different body positions on high-resolution esophageal pressure topography (HREPT) parameters. Dis Esophagus 2015;28:246-252.

23. Hollenstein M, Thwaites P, Bütikofer S, et al. Pharyngeal swallowing and oesophageal motility during a solid meal test: a prospective study in healthy volunteers and patients with major motility disorders. Lancet Gastroenterol Hepatol 2017;2:644-653.

24. Sweis R, Anggiansah A, Wong T, Kaufman E, Obrecht S, Fox M. Normative values and inter-observer agreement for liquid and solid bolus swallows in upright and supine positions as assessed by esophageal highresolution manometry. Neurogastroenterol Motil 2011;23:509-e198.

25. Wang YT, Tai LF, Yazaki E, et al. Investigation of dysphagia after antireflux surgery by high-resolution manometry: impact of multiple water swallows and a solid test meal on diagnosis, management, and clinical outcome. Clin Gastroenterol Hepatol 2015;13:1575-1583. 
26. Carlson DA, Roman S. Esophageal provocation tests: are they useful to improve diagnostic yield of high resolution manometry? Neurogastroenterol Motil 2018;30:e13321.

27. Goyal RK, Chaudhury A. Physiology of normal esophageal motility. J Clin Gastroenterol 2008;42:610-619.

28. Fonseca EK, Yamauchi FI, Tridente CF, Baroni RH. Corkscrew esopha- gus. Abdom Radiol (NY) 2017;42:985-986.

29. Prabhakar A, Levine MS, Rubesin S, Laufer I, Katzka D. Relationship between diffuse esophageal spasm and lower esophageal sphincter dysfunction on barium studies and manometry in 14 patients. AJR Am J Roentgenol 2004;183:409-413. 